\section{Origin of Bulk Nanoscale Morphology in Conducting Polymers}

\author{
Sumedh P. Surwade, Neha Manohar, and \\ Sanjeev K. Manohar* \\ Department of Chemical Engineering, University of \\ Massachusetts Lowell, Lowell, Massachusetts 01854
}

Received January 21, 2009

Revised Manuscript Received February 18, 2009

The direct one-step chemical transformation of a liquid monomer to bulk conducting polymer nanofibers has opened new opportunities in the field of energy storage and chemical/ biological sensors. ${ }^{1}$ The conducting polymer polyaniline has evolved as a prototype system in the study of this transformation, and there are now several empirical methods to convert aniline directly to bulk conducting polyaniline nanofibers. ${ }^{2-13}$ The key to broadening this phenomenon to other conducting and classical polymers would be in uncovering the mechanism of nanofiber formation. Here we report that surfaces such as the walls of the reaction vessel and/or intentionally added surfaces play a dramatic role in the evolution of nanofibrillar morphology. ${ }^{14}$ Nucleation sites on surfaces promote the accumulation of aniline dimer that reacts further to yield aniline tetramer, which (surprisingly) is entirely in form of nanofibers and whose morphology is transcribed to the bulk by a double heterogeneous nucleation mechanism. This unexpected phenomenon could form the basis of nanofiber formation in all classes of precipitation polymerization systems.

The conventional chemical oxidative polymerization of aniline using ammonium peroxydisulfate oxidant in dilute aqueous acids yields a granular polyaniline powder having very little nanoscale morphology. The reaction is characterized by an induction period that can last from a few minutes to hours depending on temperature, concentration, etc., which is followed by a rapid precipitation of a dark-green polyaniline powder. By altering the synthetic conditions during the induction period, it is possible to completely change the morphology of polyaniline from granules to nanofibers. For example, nanofibers are obtained by carrying out the polymerization at the interface of two immiscible solvents ${ }^{15}$ or by simply adding catalytic (seed) quantities of nanofibers of known composition of any kind during the polymerization (called nanofiber seeding). ${ }^{5}$ Adding aniline oligomers to the reaction ${ }^{16-18}$ or simply diluting the reaction mixture by a factor of 20 also yields polyaniline nanofibers. ${ }^{8}$ However, the nanofibers obtained in most systems look strikingly alike, i.e., a nonwoven mesh of fibers that are microns long and $30-70 \mathrm{~nm}$ in diameter, suggesting a common underlying mechanism.

We suspected that available surfaces, such as the walls of the reaction vessel, magnetic stir bar, etc., could be playing an important role in nanofiber formation. ${ }^{19}$ This was based on laser light scattering studies that showed that rodlike aggregates are formed in systems that yielded nanofibers (spherical aggregates yielded granules). ${ }^{6}$ Since these aggregates would be highly hydrophobic, we suspected that they would deposit on available surface sites and initiate polymerization from these sites. To increase the hydrophobicity of the surface, we carried out a conventional polyaniline synthesis in a glass vessel (no nanofi-

\footnotetext{
* Corresponding author: e-mail sanjeev_manohar@uml.edu; Ph 978-9343162 .
}

bers, Figure 1a, inset) containing a rolled-up sheet of commercial poly(ethylene terephthalate) (PET). The reaction rate increases significantly, and surprisingly, the morphology changes dramatically from granules to entirely nanofibers (Figure 1a). The reaction is initiated on the PET surface as evidenced by the dark blue color of in situ deposited pernigraniline film that is much thicker than corresponding films on a glass surface (control).

Conversely, it is also possible to obtain granules under conditions that typically yield nanofibers, e.g., when the reaction is carried out at $20 \times$ dilution $^{20}$ in a glass beaker. If the reaction is carried out in a stainless steel vessel, nanofiber growth is completely suppressed and only granules are obtained (Figure 1b). A stainless steel surface is mildly reactive under the acidic reaction conditions and would not permit the deposition of hydrophobic species from solution. If a PET sheet is immersed in the stainless steel vessel, nanofiber formation is restored, showing that surface effects are playing an important role in orchestrating nanofibrillar growth (Figure 1c). In contrast, a recent report describes nanofiber formation in an aniline dimer promoted reaction where no surface films are formed. ${ }^{18}$

To unequivocally elucidate the effect of surfaces in nanofiber formation, we identified a reaction vessel that would, in essence, have "no surface". We found that a flask made entirely of ice satisfies the condition in that its surface would constantly be in equilibrium with the reaction mixture and would not allow any permanent surface deposition to take place. We carried out the reaction in an ice vessel at $8 \times$ dilution and found no nanofibers (Figure 2a). Under these conditions nanofibers are readily formed in a glass vessel (Figure 2a inset), suggesting that when there is no surface to promote heterogeneous nucleation, homogeneous nucleation is the only available pathway, and this favors granular polymer growth. Importantly, when a PET sheet is immersed in the ice vessel, nanofiber formation is restored which is consistent with a change back to heterogeneous nucleation (Figure 2b).

We believe that the formation of aniline dimer on surfaces is playing a key role in bulk nanofiber formation. We reported earlier that when a small amount of aniline dimer is added during the induction period, the reaction rate increases and nanofibers are obtained. ${ }^{16}$ This is qualitatively similar to what is observed when a PET sheet is immersed in the reaction. Similar results using aniline dimer have recently been reported, ${ }^{18}$ although it is to be noted that aniline dimer is an intermediate regardless of bulk polyaniline morphology, and something strikingly different is taking place when solid aniline dimer is added to the reaction.

In a control experiment, aniline dimer alone was treated with peroxydisulfate in aqueous $1.0 \mathrm{M} \mathrm{HCl}$. Contrary to previously published results on aniline dimer oxidation, ${ }^{21-23}$ we observe a near-instantaneous formation of a blue-green powder that is analytically and spectroscopically consistent with hydrochloridedoped aniline tetramer and not polyaniline (see Supporting Information Section). ${ }^{24,25}$ The yield is quantitative based on oxidant, and the powder displays a four-probe pressed pellet electronic conductivity $\sigma_{\mathrm{RT}} \sim 10^{-2} \mathrm{~S} / \mathrm{cm}$. To our surprise, the tetramer powder is composed entirely of very long 40-80 nm diameter nanofibers (no granules, Figure 3a).

The near-instantaneous oxidative dimerization of aniline dimer coupled with what appears to be simultaneous nanofiber formation is a new phenomenon and shows that extremely short 

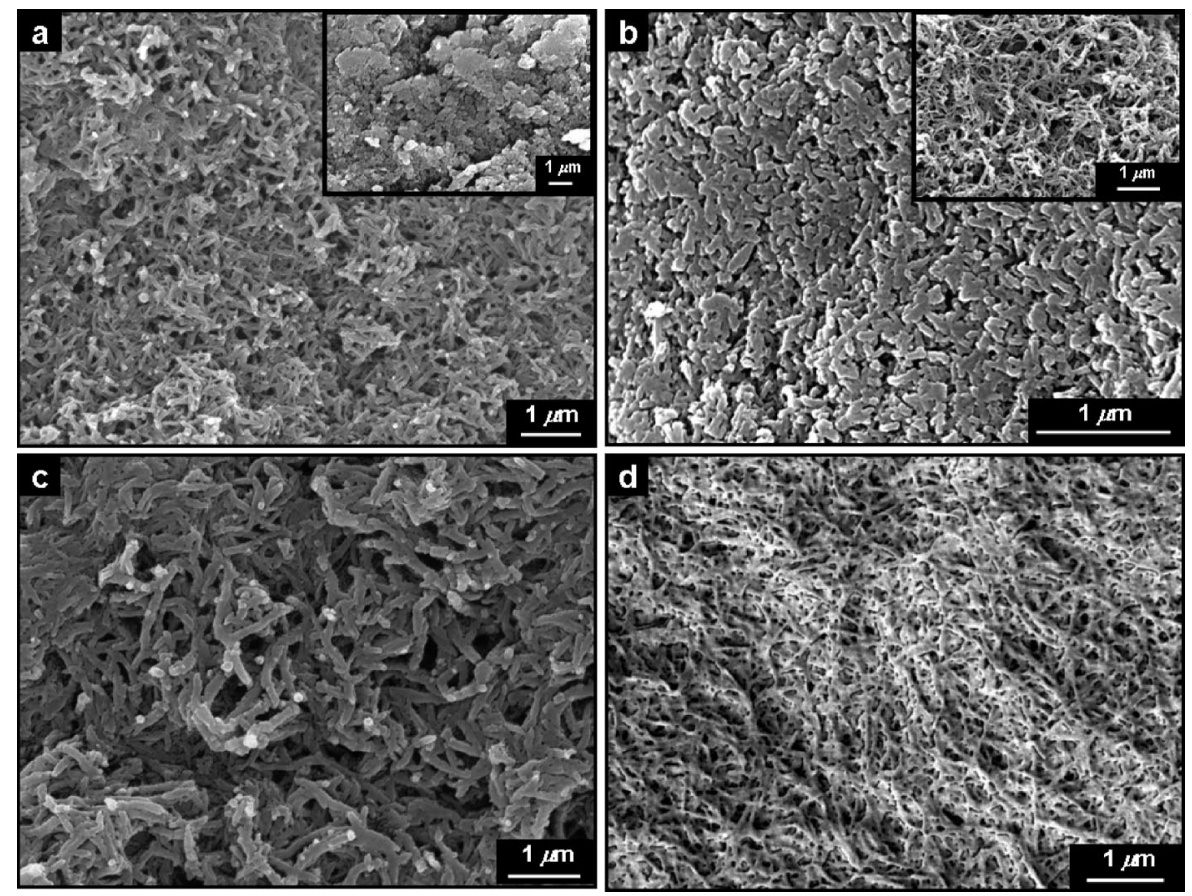

Figure 1. Scanning electron microscopy images. (a) Conventional synthesis of polyaniline $\cdot \mathrm{Cl}$ powder, at $0{ }^{\circ} \mathrm{C}$ using $0.43 \mathrm{M}$ aniline in a glass vessel containing a PET sheet. Inset: in a glass vessel (control). (b) Polyaniline ${ }^{\circ} \mathrm{Cl}$ powder synthesized at $23^{\circ} \mathrm{C}$ and $20 \times$ dilution in a stainless steel vessel. Inset: in a glass vessel (control). (c) Polyaniline $\cdot \mathrm{Cl}$ powder synthesized at $23{ }^{\circ} \mathrm{C}$ at in a stainless steel vessel containing a PET sheet. (d) Aniline tetramer nanofibers formed in situ on the surface of a PET sheet when a thin film of aniline dimer deposited on PET is immersed into aqueous $0.2 \mathrm{M}$ ammonium peroxydisulfate/ $1 \mathrm{M} \mathrm{HCl}$ solution.
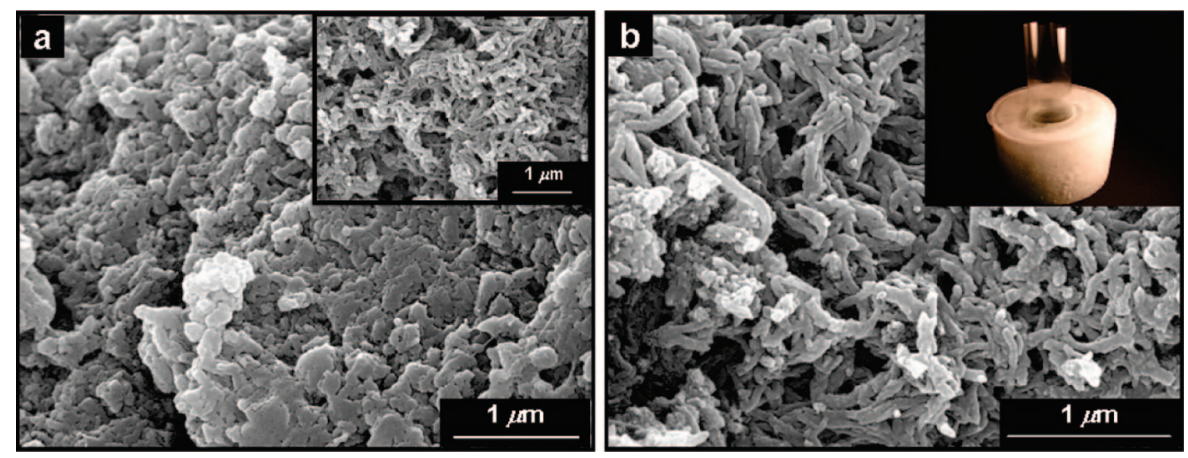

Figure 2. Scanning electron microscopy images of polyaniline $\cdot \mathrm{Cl}$ powder synthesized at $0{ }^{\circ} \mathrm{C}$ at $8 \times$ dilution using $0.054 \mathrm{M}$ aniline. (a) In an ice vessel. Inset: in a glass reaction vessel. (b) Ice reaction vessel containing a sheet of PET. Inset: image of reaction system.
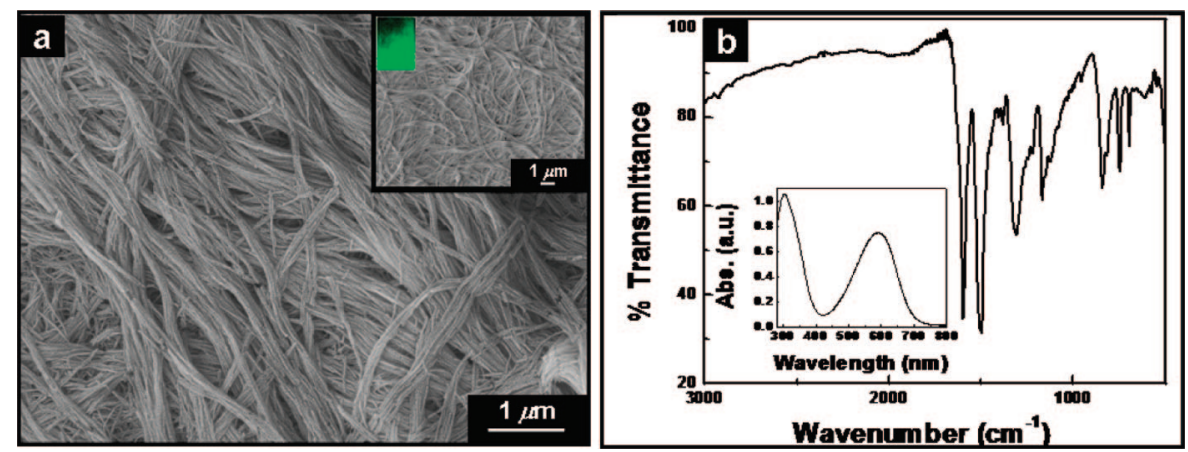

Figure 3. Characterization of aniline tetramer synthesized from aniline dimer. (a) Scanning electron microscopy image of aniline tetramer $\mathrm{Cl}$ powder. Inset: film deposited on PET. (b) Vibrational spectrum (ATR-FT/IR) of undoped aniline tetramer. Inset: UV/vis spectrum of undoped aniline tetramer (solution in NMP).

chains can yield very long nanofibers. We propose that directional polymer growth occurs when surface conditions favor the formation of tetramer nanofibers, i.e., favors high concentration of aniline dimer (clusters or aggregates). This is consistent with the redox nature of aniline coupling reactions; i.e., a fully oxidized form aniline dimer will couple with a fully reduced form to yield aniline tetramer (Scheme 1). ${ }^{26}$ The outer surface of an aniline dimer aggregate (or added insoluble aniline dimer) would be oxidized first since it is in contact with the oxidant in solution. Redox coupling would then take place with reduced 
Scheme 1. Two Pathways for the Formation of Polyaniline Nanofibers or Granules ${ }^{a}$

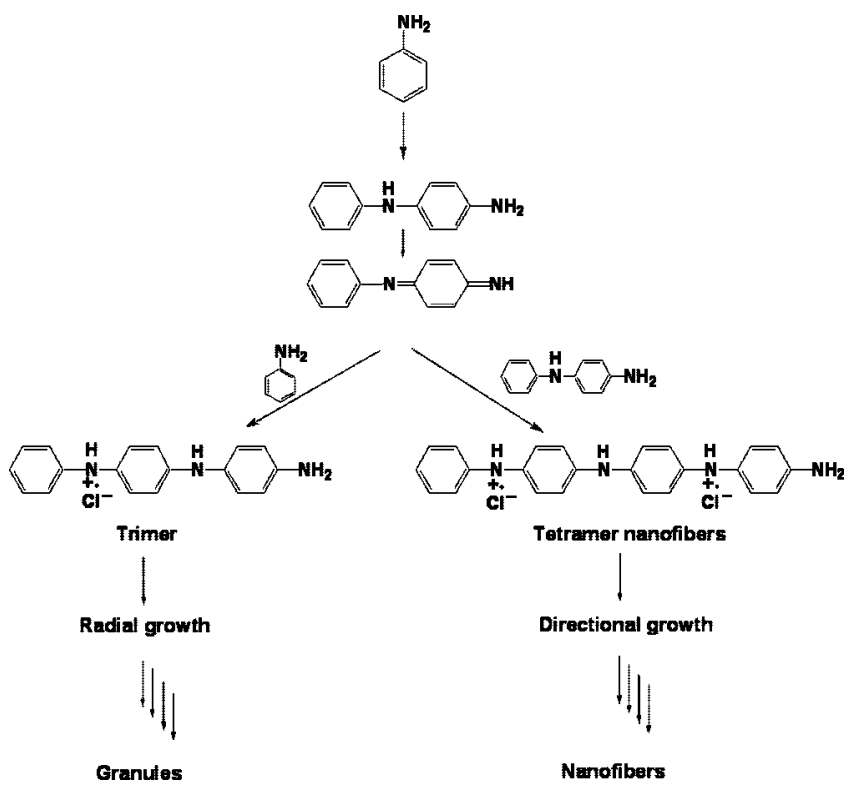

${ }^{a}$ Trimer pathway (left) leads to granules and tetramer pathway (right) leads to nanofibers.

(unreacted) dimer present in the interior of the aggregate, and the resulting heat of the reaction would squeeze out fresh unreacted aniline dimer for further reaction with oxidant in solution. This could explain why extremely long fibers of aniline tetramer are obtained in spite of its low molar mass. Once formed, however, these nanofibers would seed fresh polymer growth on their surface, resulting in bulk nanofibers. ${ }^{27}$ In contrast, granules are formed by the trimer pathway shown in Scheme 1 when aniline dimer reacts nondirectionally with aniline monomer in solution. This occurs when the surface concentration of aniline dimer is low or if there is no surface available for aniline dimer deposition.

To further underscore the importance of surface phenomena in nanofiber formation, a thin film of aniline dimer is first deposited on PET by evaporating a solution in acetone. When this film is dipped into aqueous peroxydisulfate (no aniline), the film instantly turns blue yielding a nonwoven network of nanofibers of aniline tetramer (Figure 1d). Interestingly, no polyaniline is formed, and this solid state reaction takes place entirely on the PET surface with no reaction taking place in solution. This opens a new route to surface-supported nanofiber oligoaniline films for use in a variety of device applications.

Tetramer nanofiber formation also helps explain the unusual effects of concentration, agitation, temperature, and ionic strength on bulk nanofiber formation. For example, at high aniline concentration, aniline dimer would react with aniline in solution by the trimer pathway (granules), whereas a hydrophobic PET surface would increase the surface concentration of aniline dimer to favor dimer-dimer coupling. At $20 \times$ dilution aniline dimer aggregates formed on the surface of glass or PET would have sufficient time to self-couple since the solution monomer concentration of aniline is low. Strong mechanical or magnetic stirring should disfavor nanofiber formation as it would disrupt the aggregate formation and promote dimermonomer coupling. ${ }^{28}$ Other conditions that should favor dimerdimer coupling include those that typically accelerate the reaction, e.g., low ionic strength, higher reaction temperatures, ${ }^{6}$ UV- or $\gamma$-irradiation, ${ }^{9,13}$ and ultrasonication. ${ }^{10}$ Finally, in aniline dimer promoted reactions, autocatalytic polymer growth on the surface of tetramer nanofibers would disfavor deposition on the glass reaction vessel. ${ }^{18}$

In principle, the phenomenon of the intermediacy of nascent oligomeric nanofibers in orchestrating bulk nanofiber formation could be extended to all classes of conducting polymers. For example, it provides a mechanistic rationale for why nanofiber seeding using $\mathrm{V}_{2} \mathrm{O}_{5}$ nanofibers has been successfully used to synthesize nanofibers of polyaniline, polypyrrole, PEDOT, and polythiophene, ${ }^{5,29-31}$ i.e., $\mathrm{V}_{2} \mathrm{O}_{5}$ nanofiber catalyzes the formation of the fibrillar oligomeric species on its surface. It also accounts for the polypyrrole and polythiophene nanofibers formed when the reaction is seeded with small quantities of their corresponding dimers.

The above results offer a strikingly different mechanism for nanofiber formation compared to classical homogeneous/ heterogeneous nucleation based approaches. For example, in the classical model, nanofibers are formed because of homogeneous nucleation (solution), and subsequent heterogeneous nucleation on their surface is believed to result in loss of directionality leading to granules. In contrast, polyaniline nanofiber formation appears to proceed by a double heterogeneous nucleation process, i.e., the first taking place on existing surfaces yielding nascent nanofibers and a second on the surface of these nascent nanofibers in which the morphology is rapidly transcribed across multiple length scales to the bulk precipitate. This is similar to what we have observed in nanofiber seeding and is consistent with the autocatalytic nature of polyaniline growth. The double heterogeneous nucleation process for nanofiber formation bears a qualitative similarity with unusual double nucleation mechanism of deoxygenated sickle cell hemoglobin fibers. ${ }^{32}$

Acknowledgment. We gratefully acknowledge funding from the University of Massachusetts Lowell's MTC-funded Nanomanufacturing Center of Excellence (NCOE), NSF-funded Center for High-rate Nanomanufacturing (CHN), NSF award \#0425826.

Supporting Information Available: Synthetic procedure and characterization data on aniline tetramer. This material is available free of charge via the Internet at http://pubs.acs.org.

\section{References and Notes}

(1) Li, D.; Huang, J.; Kaner, R. B. Acc. Chem. Res., ACS ASAP.

(2) Chiou, N.-R.; Lu, C.; Guan, J.; Lee, L. J.; Epstein, A. J. Nat. Nanotechnol. 2007, 2, 354-357.

(3) Huang, J.; Kaner, R. B. Angew. Chem., Int. Ed. 2004, 43, 5817-5821.

(4) Huang, J.; Kaner, R. B. Chem. Commun. 2006, 367-376.

(5) Zhang, X.; Goux, W. J.; Manohar, S. K. J. Am. Chem. Soc. 2004, $126,4502-4503$.

(6) Zhang, X.; Kolla, H. S.; Wang, X.; Raja, K.; Manohar, S. K. Adv. Funct. Mater. 2006, 16, 1145-1152.

(7) Zhang, X.; Manohar, S. K. Chem. Commun. 2004, 20, 2360.

(8) Chiou, N.-R.; Epstein, A. J. Adv. Mater. 2005, 17, 1679-1683.

(9) Pillalamarri, S. K.; Blum, F. D.; Tokuhiro, A. T.; Story, J. G.; Bertino, M. F. Chem. Mater. 2005, 17, 227-229.

(10) Jing, X.; Wang, Y.; Wu, D.; She, L.; Guo, Y. J. Polym. Sci., Part A: Polym. Chem. 2005, 44, 1014-1019.

(11) Li, J.; Zhu, L.; Luo, W.; Liu, Y.; Tang, H. J. Phys. Chem. C 2007, $111,8383-8388$

(12) Wang, Y.; Jing, X. J. Phys. Chem. B 2008, 112, 1157-1162.

(13) Li, J.; Tang, H.; Zhang, A.; Shen, X.; Zhu, L. Macromol. Rapid Commun. 2007, 28, 740-745.

(14) Manohar, S. K.; MacDiarmid, A. G.; Epstein, A. J. Synth. Met. 1991, 41, 711-714.

(15) Huang, J.; Virji, S.; Weiller, B. H.; Kaner, R. B. J. Am. Chem. Soc. 2003, 125, 314-315.

(16) Manohar, S. K.; Zhang, X.; Wu, A.; Kolla, H. Polym. Prepr. (Am. Chem. Soc., Div. Polym. Chem.) 2004, 45, 587.

(17) Li, W.; Wang, H.-L. J. Am. Chem. Soc. 2004, 126, 2278-2279. 
(18) Tran, H. D.; Wang, Y.; D’Arcy, J. M.; Kaner, R. B. ACS Nano 2008, 2, 1841-1848.

(19) Maeda, S.; Armes, S. P. J. Mater. Chem. 1994, 4, 935-942.

(20) Reaction mixture diluted 20 times with aqueous $1.0 \mathrm{M} \mathrm{HCl}$ vs conventional synthesis.

(21) Sun, Q.; Deng, Y. Eur. Polym. J. 2008, 44, 3402-3408.

(22) Dias, H. V. R.; Wang, X.; Rajapakse, R. M. G.; Elsenbaumer, R. L. Chem. Commun. 2006, 976-978.

(23) Green, A. G.; Woodhead, A. E. J. Chem. Soc., Trans. 1910, 97, 23882403.

(24) Kulszewicz-Bajer, I.; Rozalska, I.; Kurylek, M. New J. Chem. 2004, $28,669-675$.

(25) Zhang, W. J.; Feng, J.; MacDiarmid, A. G.; Epstein, A. J. Synth. Met. 1997, 84, 119-120.
(26) Kolla, H. S.; Surwade, S. P.; Zhang, X.; MacDiarmid, A. G.; Manohar, S. K. J. Am. Chem. Soc. 2005, 127, 16770-16771.

(27) Liang, L.; Liu, J.; Windisch, C. F., Jr.; Exarhos, G. J.; Lin, Y. Angew. Chem., Int. Ed. 2002, 41, 3665-3668.

(28) Li, D.; Kaner, R. B. J. Am. Chem. Soc. 2006, 128, 968-975.

(29) Zhang, X.; MacDiarmid, A. G.; Manohar, S. K. Chem. Commun. 2005, $42,5328-5330$.

(30) Zhang, X.; Manohar, S. K. J. Am. Chem. Soc. 2004, 126, 12714.

(31) Zhang, X.; Surwade, S. P.; Dua, V.; Bouldin, R.; Manohar, N.; Manohar, S. K. Chem. Lett. 2008, 37, 526-527.

(32) Rotter, M. A.; Kwong, S.; Briehl, R. W.; Ferrone, F. A. Biophys. J. 2005, 89, 2677-2684.

MA900141G 